\title{
The association of liver function and quality of life of patients with liver cancer
}

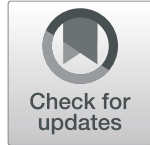

Leung Li ${ }^{1}$, Frankie Mo ${ }^{1}$, Edwin P. Hui ${ }^{1}$, Stephen L. Chann ${ }^{1}$, Jane Koh ${ }^{1}$, Nelson L. S. Tang ${ }^{3}$, Simon C. H. Yu ${ }^{4}$ and Winnie $Y e o^{1,2^{*}}$

\begin{abstract}
Background: Quality of life (QOL) assessments with the European Organization for Research and Treatment of Cancer (EORTC) QLQ-C30, QLQ-HCC18, C30 and HCC18 index scores have been shown to be prognostic factors for overall survival (OS) in patients with hepatocellular carcinoma (HCC), independent of disease stage and liver function. Liver function parameters (including bilirubin, albumin, international normalized ratio [INR], Child-Pugh class, ALBI grade, MELD, alkaline phosphatase [ALP]-to-platelet ratio, albumin-to-ALP ratio) have also been found to be independent prognostic factors for OS in HCC patients. There has been scanty data on whether QOL and baseline liver function per se are correlated in HCC patients. This study investigates the correlations between baseline QOL data and liver function variables in HCC patients.
\end{abstract}

Methods: From 2007 to 2011, 517 patients were enrolled. Baseline QOL was assessed at diagnosis using the EORTC QLQ-C30 and QLQ-HCC18; thereafter C30 and HCC18 index scores were derived. Clinical and laboratory data were collected. For liver function assessment, Child-Pugh class, ALBI grade, MELD, ALP-to-platelet ratio and albumin-toALP ratio were derived. Correlation analyses were performed between QOL and liver function data.

Results: Complete QOL data were available in 472 HCC patients. After adjusting for clinical variables, significant correlations were found between QOL (QLQ-C30 and QLQ-HCC18) and dichotomized liver function variables (including Child-Pugh class, ALBI grade and the presence of ascites). It was demonstrated that QOL had significant and potentially clinically important correlations with continuous liver function variables (albumin, bilirubin, ALP and albumin-to-ALP ratio), with the highest Spearman's rank correlation coefficient (rho) exceeding 0.4. HCC18 and C30 index scores were also significantly correlated with these liver function variables. HCC18 index score, which had rho up to 0.37 , generally performed better than C30 index score, which had rho up to 0.33 .

Conclusions: In HCC patients, baseline QOL assessment (using EORTC QLQ-C30, QLQ-HCC18, C30 index-score or HCC18 index-score) is significantly correlated with liver function. Based on the findings of this study, future trials are warranted to assess whether treatment to enhance liver function could improve HCC patients' QOL.

Keywords: Hepatocellular carcinoma, Health related quality of life, EORTC QLQ-C30, QLQ-HCC18, C30 index-score, HCC18 index-score, Hepatic function, Correlation, Child-Pugh, MELD, Albumin to alkaline phosphatase ratio, Alkaline phosphatase to platelet ratio, ALBI

\footnotetext{
* Correspondence: winnieyeo@cuhk.edu.hk

'Department of Clinical Oncology, Prince of Wales Hospital, Faculty of

Medicine, The Chinese University of Hong Kong, Shatin, NT, Hong Kong SAR

${ }^{2}$ State Key Laboratory in Oncology in South China, Prince of Wales Hospital,

Faculty of Medicine, The Chinese University of Hong Kong, Shatin, Hong

Kong SAR

Full list of author information is available at the end of the article
}

(c) The Author(s). 2019 Open Access This article is distributed under the terms of the Creative Commons Attribution 4.0 International License (http://creativecommons.org/licenses/by/4.0/), which permits unrestricted use, distribution, and reproduction in any medium, provided you give appropriate credit to the original author(s) and the source, provide a link to the Creative Commons license, and indicate if changes were made. The Creative Commons Public Domain Dedication waiver (http://creativecommons.org/publicdomain/zero/1.0/) applies to the data made available in this article, unless otherwise stated. 


\section{Background}

Liver cancer ranked the fifth most common cancer in the world, with 782,451 new cases per year in 2012. It was also the second leading cause of cancer death worldwide, responsible for 745,533 deaths in 2012. The ratio of mortality to incidence was 0.95 , signifying its very aggressive nature and poor prognosis [1]. Screening population at risk for HCC by regular ultrasonography and alpha fetoprotein (AFP) level has been proven in one study to improve rate of early detection and increase the chances of curative treatment and survival [2]. However, for most parts of the world, only opportunistic screening is offered to patients with chronic liver diseases [3]. Various management guidelines for HCC are available, these include the Barcelona Clinic Liver Cancer staging classification and treatment approach for HCC (BCLC) [4, 5], Asia-Pacific Association for the Study of the Liver guidelines on the management of HCC (APASL) [6], European Association For The Study Of The Liver-European Organization For Research And Treatment Of Cancer clinical practice guidelines on management of HCC (EASL) [7], and the American Association for the Study of Liver Diseases guidelines for the treatment of HCC (AASLD) [8]. These guidelines unanimously recommend that preserved liver function is an important pre-requisite to deliver effective treatment to HCC patients.

Most hepatocellular carcinomas (HCC) arise in patients with cirrhosis. The most frequent etiologies of HCC in western countries have been chronic hepatitis C virus (HCV) infection and alcoholic liver disease [9-13], while in Asian countries, the main etiological factor has been chronic hepatitis B virus (HBV) infection $[9,10$, 14]. Regardless of etiology, patients with $\mathrm{HCC}$ are frequently challenged by impairment of liver function as a result of chronic liver disease and cirrhosis in addition to liver tumor burden. Inadequate liver functional reserve creates difficulty in clinical management and negatively affect prognosis.

Liver function has been demonstrated to be associated with overall survival (OS) in patients with HCC. Specifically, parameters that have been reported to be independent prognostic factors in HCC include bilirubin $[15,16]$, albumin [16], international normalized ratio (INR) [17], alkaline phosphatase (ALP) [18], Child-Pugh classification $[19,20]$, albumin-bilirubin (ALBI) grade [21, 22], Model for End-stage Liver Disease (MELD) [23], ALP-to-platelet ratio [24] and albumin-to-ALP ratio [17, 18, 25].

QOL in HCC patients is a complex issue affected by medical, psychological, social and spiritual factors [26]. While symptoms arising from HCC tumor per se, the underlying chronic liver disease and cirrhosis, as well as therapeutic intervention for HCC might affect patients' QOL, other aspects including socio-spiritual support, patient's coping skill, cultural background and health literacy could also influence QOL. Various instruments have been developed to measure QOL in HCC patients.

The European Organization for Research and Treatment of Cancer (EORTC) QLQ-C30 and Spitzer Quality of Life Index have been found to be prognostic of OS in patients with advanced stage HCC [27-29]. Subsequently, the EORTC QLQ-HCC18, a QOL instrument specifically designed to address issues faced by HCC patients, has also been found to be prognostic for OS in unselected HCC patients, independent of HCC stage and liver function [30]. More recently, in an attempt to simplify the interpretation of results obtained from the various functional domains and symptoms/items scores in the EORTC QLQ-C30 and QLQ-HCC18 assessment, we reported two scoring indices, namely the C30 index-score and HCC18 index-score. These are two single summative scores representing QLQ-C30 and QLQ-HCC18 respectively and they facilitate survival analysis. These have been demonstrated to be highly significant factors for OS [30]. On the other hand, Functional Assessment of Cancer Therapy - General (FACT-G) has been evaluated but has not been reported to be prognostic of OS in HCC patients [31].

Although functional impairment of the hepatic organ commonly occurs in HCC patients, there is very limited data on whether baseline liver function and QOL are correlated in HCC patients. We hypothesized that QOL in HCC patients is correlated with the status of their liver function. We, therefore, attempt to assess the correlation between liver function and QOL in HCC patients.

\section{Methods}

\section{Patients and methods}

The study was approved by the Joint Chinese University of Hong Kong - New Territories East Cluster Clinical Research Ethics Committee. From January 2007 to December 2011, all patients with newly diagnosed HCC who attended the Joint Hepatoma Clinic of the Prince of Wales Hospital were invited to this study. Informed written consents were obtained from all participants.

The eligibility criteria included age 18 years or above; newly diagnosed HCC established either by histology, or the combination of radiological and biochemical findings (hypervascular hepatic lesion and elevated AFP $\geq 400 \mu \mathrm{g} / \mathrm{L}$, or typical pattern in 2 radiological modalities by ultrasonography, multiphasic computed tomography, magnetic resonance imaging or angiography); treatment-naïve for $\mathrm{HCC}$, ability to read and comprehend Chinese. The exclusion criteria included history of other malignancies, encephalopathy or cognitive impairment. 


\section{QOL assessment}

The Chinese version of EORTC QLQ-C30 [32] and QLQ-HCC18 [33] questionnaires were used to assess patients' baseline QOL on the same day upon entering the study. C30 and HCC18 index-scores were calculated as previously published [30]. Detailed descriptions of these are listed in Table 1.

\section{Clinical factors}

Demographic, clinical and laboratory data were also collected on the day of study entrance. Since there was no prior information on the relationship between QOL and liver function in HCC patients, we explored a broad array of liver function variables in the analyses. These included albumin, ALP, alanine transaminase (ALT), bilirubin, INR and platelet counts. Presence or absence of ascites was noted. Child-Pugh class, MELD, ALBI grade, albumin-to-ALP ratio and ALP-to-platelet ratio were derived (see Table 2).

\section{Statistical analysis}

Standard descriptive analyses were performed to assess sample characteristics. The following liver function assessments based on stratified scoring systems were dichotomized into normal versus abnormal groups: Child-Pugh class ('A' versus 'B and C'), ALBI grade (' 1 ' versus ' 2 and 3 '), MELD grade (' 1 ' versus ' 2 and 3 '), respectively. Univariate logistic regressions were used to evaluate the correlations between dichotomized liver function variables and continuous QOL factors. To control for clinical factors, baseline clinical variables including age, gender, performance status, liver biochemistry, AFP level, presence of cirrhosis, etiology of cirrhosis, tumor stage and planned treatment, together with all significant QOL variables obtained from univariate logistic model were entered into the multivariate logistic regressions with stepwise selection. For all logistic regressions, odds ratios (OR) were calculated with odds of patients belonging to abnormal liver function group against normal liver function group. Ninety-five percent confidence intervals $(95 \% \mathrm{CI})$ of OR were also calculated. Since the QOL data were not normally distributed, the correlations between continuous liver function parameters (in natural logarithm) and the QOL data were assessed using Spearman's rank correlation analysis. A $p$-value of less than 0.05 was accepted as statistically significant. Further, we defined Spearman's rank correlation outcomes with coefficient (rho) of $\geq 0.3$ or $\leq-0.3$ as potentially clinically important [34-37]. The statistical analyses were performed using statistical software (SAS version 9.3; SAS institute, Cary, NC, USA).

\section{Sample size estimation}

Assuming Spearman's rho between QOL and liver function variables to be at least 0.3 , with two sided alpha-level of 0.05 and power of 0.9 , the required sample size was 133 patients [38]. We aimed to obtain C30 and HCC18 index-scores for

Table 1 Quality of life instruments used in the study

Quropean Organization for Research and Treatment
of Cancer QLQ-C30 (EORTC QLQ-C30) [32]

European Organization for Research and Treatment of Cancer QLQ-HCC18 (EORTC QLQ-HCC18) [33]

C30 index score [30]
Description

EORTC QLQ-C30 is a general cancer instrument containing multiple items, measured in multiple-point Likert scales, that reflect the multidimensionality of QOL construct [32]. It includes five functional domains (physical, role, cognitive, emotional and social), three symptom domains (fatigue, pain, nausea and vomiting), and a global health and QOL domain. Six single items assess common symptoms in cancer patients (dyspnea, appetite loss, sleep disturbance, constipation and diarrhea) and financial problem. All scales and domains are transformed to scores ranging from 0 to 100. A lower score for a functional or global $\mathrm{QOL}$ scale reflects a poorer functioning level or global $\mathrm{QOL}$, while a lower score for a symptom/problem scale reflects less symptoms/problem (better QOL).

EORTC QLQ-HCC18 includes eighteen multiple item scales organized into six domains (fatigue, body image, jaundice, nutrition, pain and fever) and two items (abdominal swelling and sex life) [33]. All scales are grouped and transformed to score ranging from 0 to 100. A lower score represents a less severe symptom/problem (better QOL). EORTC QLQ-HCC18 is used together with EORTC QLQ-C30.

$\Sigma$ [(100-Physical functioning), (100-Role functioning), (100-Emotional functioning), (100-Cognitive functioning), (100-Social functioning), (100-global QOL), scores of Fatigue, Nausea and vomiting, Pain, Dyspnoea, Insomnia, Appetite loss, Constipation, Diarrhea, Financial Diffculty] $\div 15$.

(remarks: a lower score represents a less severe symptom/problem) 
Table 2 Scoring systems for liver function used in the study

\begin{tabular}{ll}
\hline ALBI score & $-0.085 \times$ albumin $+0.66 \times \log$ bilirubin \\
& $($ remarks: albumin in $\mathrm{g} / \mathrm{L}$, bilirubin in $\mu$ mol/L) \\
MELD score & {$[9.57 \times \ln ($ Creatinine $\div 88.4)]+[3.78 \times \ln ($ Bilirubin $\div 17.1)]+[11.2 \times \ln (I N R)]+6.43$} \\
& $($ remarks: bilirubin in umol/L, creatinine in umol/L) \\
Albumin-to-ALP ratio & albumin $\div$ ALP \\
& (remark: albumin in $\mathrm{g} / \mathrm{L}, \mathrm{ALP}$ in iu/L) \\
ALP-to-platelet ratio & ALP $\div$ platelet \\
& (remarks: ALP in iu/L, platelet count in $\left.10^{9} / \mathrm{L}\right)$ \\
\hline
\end{tabular}

ALBI Albumin-Bilirubin, MELD Model for End-stage Liver Disease, QOL Quality of life, In natural logarithm

all analyzed patients. This required all patients to have all QOL questions answered. For this reason complete-case analysis would be preferred in the study in order to calculate index-scores accurately. To minimize the impact of complete-case analysis, we set the final target sample size to be three times the original sample size (399 patients).

\section{Results}

\section{Patient characteristics}

Five hundred and seventeen patients were consented, amongst whom 472 (91\%) had complete QOL data and were included for analysis. Tables 3 and 4 showed the clinical characteristics and baseline QOL data of these patients. The median age at diagnosis was 60. Ninety-six percent had Eastern Cooperative Oncology Group (ECOG) performance status of 0 or 1 . Sixty-eight percent of all patients were of Child-Pugh class A. Fifty-nine percent had cirrhosis. HBV infection was present in $82 \%$, while $6 \%$ had HCV infection. One hundred and eight (23\%) patients had extra-hepatic metastasis. 152 patients (32\%) had portal vein thrombosis.

\section{Correlations between QOL and liver biochemistries}

Albumin level had significant correlations with C30 index-score, HCC18 index-score, and majority of scales in QLQ-C30 and QLQ-HCC18 (see Table 4). A higher albumin level was correlated with a better QOL. Among these correlations, six were considered to be potentially clinically important, namely QLQ-C30 'physical functioning, 'role functioning, 'fatigue', QLQ-HCC18 'fatigue, 'abdominal swelling' and HCC18 index-score. Figure 1 shows the scatter plot of HCC18 index-score against albumin level.

Bilirubin level had significant correlations with C30 index-score, HCC18 index-score, majority of scales in QLQ-C30 and QLQ-HCC18 (see Table 4). The lower the bilirubin level, the better the QOL. Two correlations, QLQ-HCC18 'abdominal swelling' and HCC18 indexscore, were potentially clinically important. Figure 2 shows the scatter plot of HCC18 index-score versus bilirubin level.

INR level had significant correlations with C30 indexscore, HCC18 index-score, most scales in QLQ-C30 and all the eight scales in QLQ-HCC18 (Table 4). Lower INR level was correlated with better QOL. A significant and relatively stronger correlation was seen with QLQ-C30 'fatigue'; however, the Spearman's rho was only 0.25 and thus, there was no correlation identified to be potentially clinically important.

ALP level had significant correlations with C30 indexscore, HCC18 index-score, most scales in QLQ-C30 and all QLQ-HCC18 scales (Table 4). Patients with lower ALP had better QOL. Five correlations were potentially clinically important: HCC18 index-score, C30 index-score, QLQ-HCC18 nutrition, QLQ-C30 'fatigue' and 'role functioning'. Figure $3 \mathrm{a}$ and $\mathrm{b}$ show the scatter plots of C30 and HCC18 index-scores against ALP level respectively.

ALT level had significant correlations with C30 indexscore, HCC18 index-score, a few scales in QLQ-C30 and QLQ-HCC18 (Table 4). However, none of these correlations was considered to be potentially clinically important.

\section{Correlation between QOL and albumin-to-ALP ratio}

Albumin-to-ALP ratio had significant correlations with C30 index-score, HCC18 index-score, majority of scales in QLQ-C30 and all eight scales in QLQ-HCC18 (see Table 4). A better albumin-to-ALP ratio was associated with a better QOL. Ten potentially clinically important correlations were identified: HCC18 index-score, C30 index-score, QLQ-HCC18 'nutrition,' 'abdominal swelling, 'fatigue,' 'body image, QLQ-C30 'fatigue,' role functioning,' 'physical functioning' and 'appetite loss'. Figure $4 \mathrm{a}$ and b show the scatter plots of C30 and HCC18 index-scores against albumin-to-ALP ratio respectively.

\section{Correlation between QOL and ALP-to-platelet ratio}

ALP-to-platelet ratio had significant correlations with C30 index-score, HCC18 index-score, a few scales in QLQ-C30 and QLC-HCC18 (see Table 4). However, none of these correlations was high enough to be regarded as potentially clinically important.

\section{Correlation between QOL and child-Pugh class}

Thirty-two percent of patients were in Child's class B or $\mathrm{C}$, the rest were in Child's class A (see Table 3). There were significant correlations between Child's class and 
Table 3 Baseline characteristics of in the 472 HCC patients

\begin{tabular}{|c|c|c|c|}
\hline Variable & $\mathrm{N}$ & $\%$ & Mean \pm SD \\
\hline \multicolumn{4}{|l|}{ Clinical data } \\
\hline Age $<=65$ & 328 & 69 & $60 \pm 12$ \\
\hline Male gender & 419 & 89 & \\
\hline \multicolumn{4}{|l|}{ ECOG } \\
\hline 0 & 144 & 31 & \\
\hline 1 & 299 & 63 & \\
\hline$\geq 2$ & 29 & 6 & \\
\hline Cirrhosis (radiological) & 278 & 59 & \\
\hline \multicolumn{4}{|l|}{ Tumor morphology } \\
\hline Uninodular & 122 & 26 & \\
\hline Multinodular & 143 & 30 & \\
\hline Diffuse & 207 & 44 & \\
\hline Extrahepatic metastasis (nodal or distant) & 108 & 23 & \\
\hline Portal vein thrombosis & 152 & 32 & \\
\hline Hepatitis B surface antigen + & 386 & 82 & \\
\hline Hepatitis C antibody + & 30 & 6 & \\
\hline a-feto protein $\geq 200 \mathrm{mg} / \mathrm{ml}$ & 250 & 53 & \\
\hline ALBI score & 472 & & $-2.29 \pm 1.33$ \\
\hline Grade 1 & 155 & 32.8 & \\
\hline Grade 2 & 265 & 56.2 & \\
\hline Grade 3 & 52 & 11.0 & \\
\hline MELD score & 472 & & $9.08 \pm 4.05$ \\
\hline Grade $1(<10)$ & 319 & 67.6 & \\
\hline Grade 2 (10-14) & 109 & 21.4 & \\
\hline Grade 3 (> 14) & 52 & 11.0 & \\
\hline \multicolumn{4}{|l|}{ Child-Pugh class } \\
\hline A & 319 & 67.6 & \\
\hline B & 130 & 27.5 & \\
\hline C & 23 & 4.9 & \\
\hline Albumin & 472 & 100 & $37.4 \pm 15.2$ \\
\hline Bilirubin & 472 & 100 & $32.8 \pm 45.7$ \\
\hline INR & 472 & 100 & $1.15 \pm 0.17$ \\
\hline ALP & 472 & 100 & $193.4 \pm 155.8$ \\
\hline ALT & 472 & 100 & $77.3 \pm 69.4$ \\
\hline Albumin-to-ALP ratio & 472 & 100 & $0.29 \pm 0.22$ \\
\hline ALP-to-platelet ratio & 472 & 100 & $1.20 \pm 1.19$ \\
\hline Presence of ascites & 122 & 25.8 & \\
\hline Hemoglobin $<10 \mathrm{~g} / \mathrm{dL}$ & 27 & 6 & \\
\hline White cell count $>10 \times 10^{9} / \mathrm{L}$ & 64 & 14 & \\
\hline Platelet count $<100 \times 10^{9} / \mathrm{L}$ & 33 & 7 & \\
\hline \multicolumn{4}{|l|}{ 1st line Treatment } \\
\hline Surgical treatment & 54 & 12 & \\
\hline Local ablative therapies & 29 & 6 & \\
\hline Trans-arterial therapies & 116 & 25 & \\
\hline
\end{tabular}

Table 3 Baseline characteristics of in the 472 HCC patients (Continued)

\begin{tabular}{lcc}
\hline $\begin{array}{l}\text { Systemic therapies } \\
\text { Best supportive care alone }\end{array}$ & 91 & 19 \\
\hline EORTC European Organization for Research and Treatment of Cancer, ECOG & 38 \\
Eastern Cooperative Oncology Group, ALBI Albumin-bilirubin, MELD Model for \\
End-stage Liver Disease, INR international normalized ratio, ALP alkaline
\end{tabular}

QOL variables in univariate logistic regressions (see Tables 5). Patients with worse C30 index-score, HCC18 index-score, as well as worse QOL scores in majority of scales in QLQ-C30 and QLQ-HCC18 were more likely to be in Child's class B or C $(p<0.01)$. After adjusting for clinical variables, QOL remained significantly correlated with Child's class (see Table 6).

\section{Correlation between QOL and ALBI grade}

Three hundred and seventeen patients (67\%) were classified as ALBI grades 2-3, 155 (33\%) as ALBI grade 1 (Table 3). QOL had significant correlations with ALBI grade (see Table 5). Patients with worse C30 index-score, HCC18 index-score, as well as worse scores in majority of scales in QLQ-C30 and all the eight scales in QLQ-HCC18 were more likely to be of ALBI grade 2 or 3 ( $p$ < 0.03). After adjusting for clinical variables, QOL remained significantly correlated with ALBI grade (see Table 6).

\section{Correlation between QOL and the presence of ascites}

One hundred and twenty two patients (26\%) presented with ascites at diagnosis (Table 3). QOL was significantly correlated with the presence of ascites (see Table 5). Patients with worse C30 index-score, HCC18 index-score, as well as worse QOL scores in majority of scales in QLQ-C30 and QLQ-HCC18 were more likely to have ascites $(p<0.05)$. After adjusting for clinical variables, QOL remained significantly correlated with ALBI grade (see Table 6).

\section{Correlation between QOL and MELD}

One hundred and sixty one patients (32.4\%) were of MELD grade 2 or 3, while $319(67.6 \%)$ were of grade 1 (see Table 3). There were significant correlations between MELD grade and QOL variables in univariate logistic regressions (see Tables 5). Patients with worse C30 index-score, HCC18 index-score, as well as worse scores in majority of scales in QLQ-C30 and QLQ-HCC18 were more likely to be of MELD grade 2 or $3(\mathrm{p}<0.05)$. However, after controlling for clinical variables, no QOL variable was significantly correlated with MELD grade (see Table 6). 


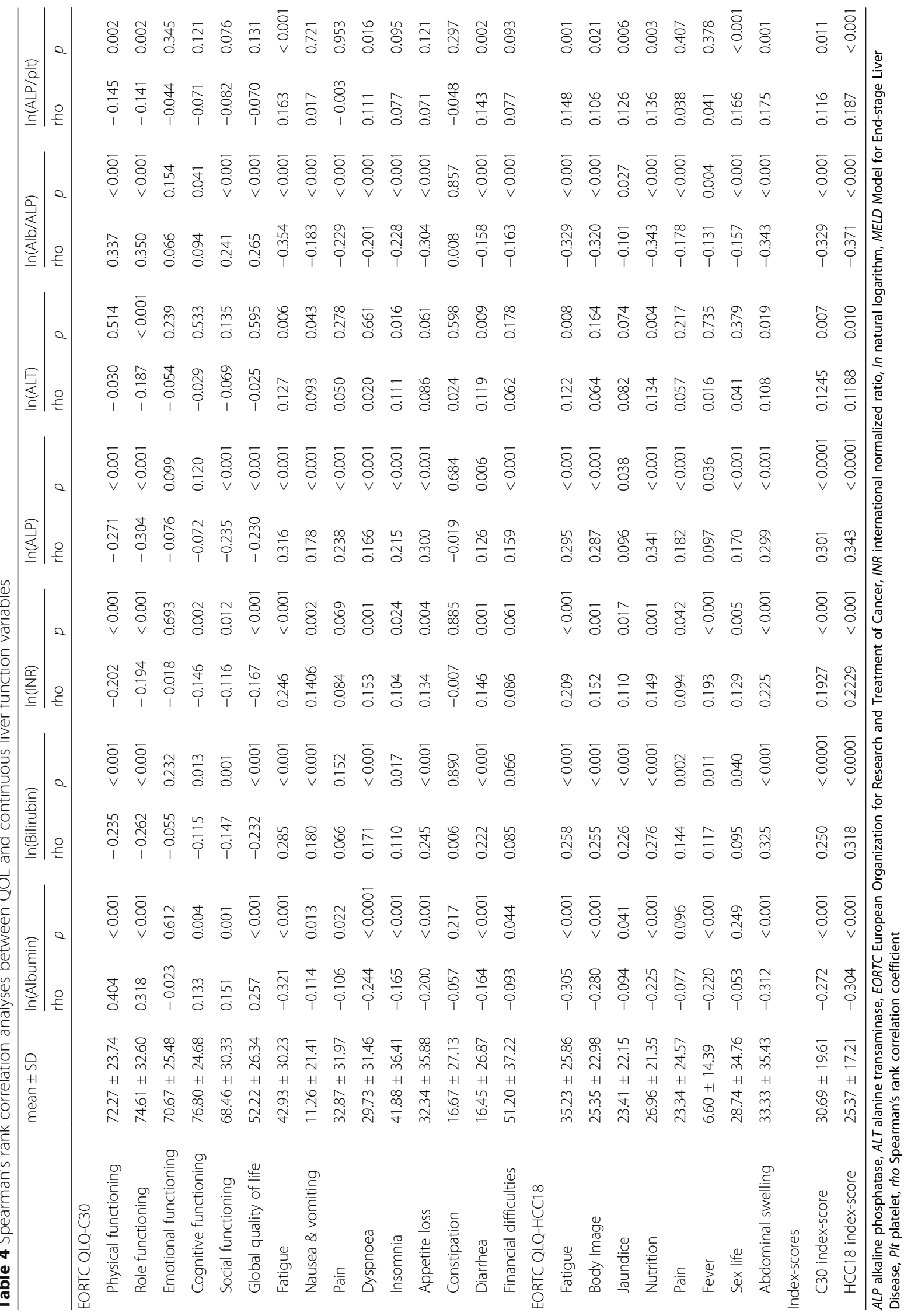




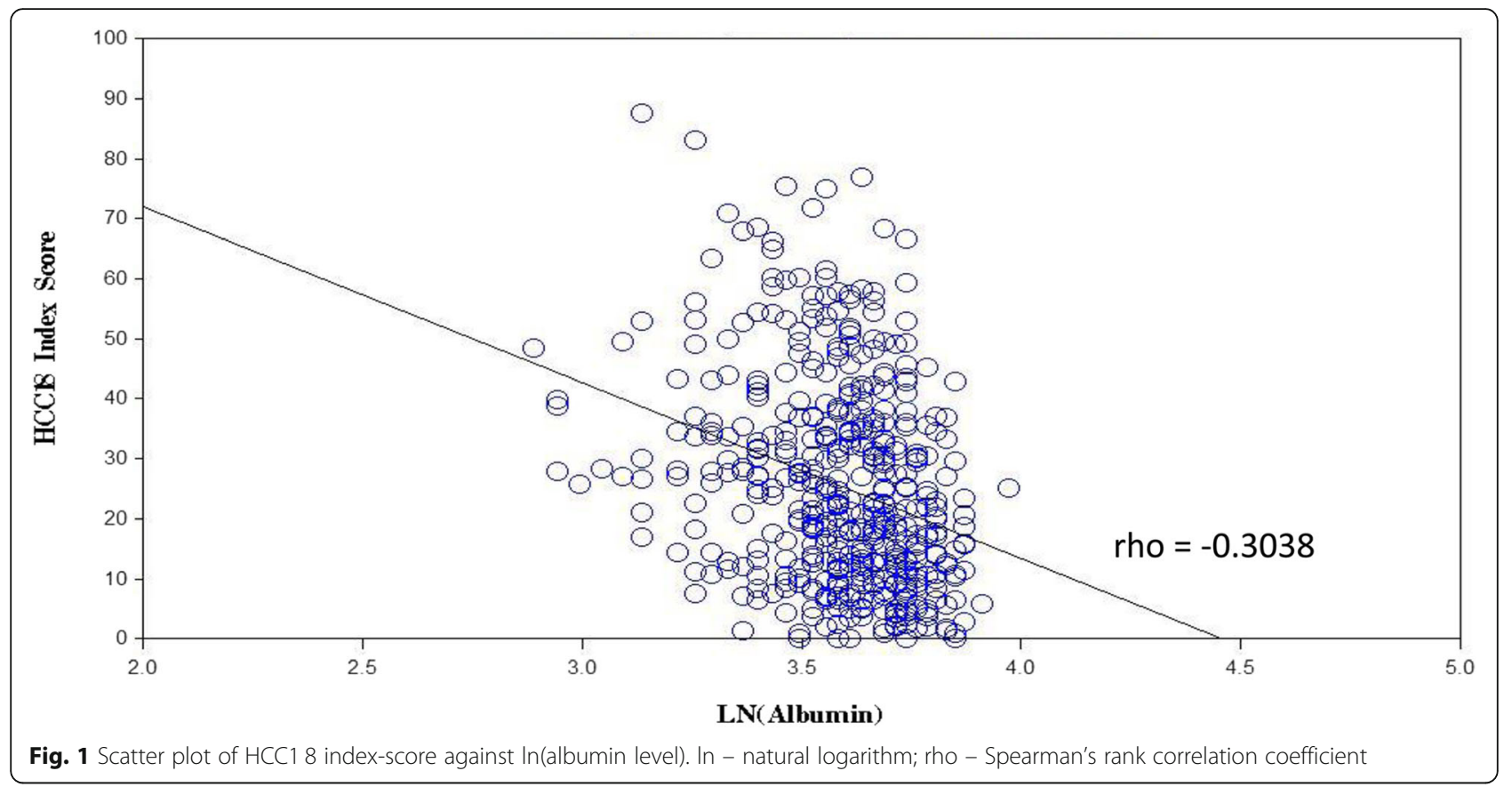

\section{Discussion}

This is the first report on the correlation between QOL and baseline liver function in patients with HCC. QOL assessments with EORTC QLQ-C30 and QLQ-HCC18 had significant correlations with most of the continuous liver function parameters evaluated. The correlations with levels of albumin, bilirubin, ALP and albuminto-ALP ratio were potentially clinically important (where Spearman's rho were $\geq 0.3$ or $\leq-0.3$ ). After adjusting for clinical variables, QOL was also demonstrated to have significant correlations with dichotomized liver function factors, including Child's class, ALBI grade and the presence of ascites,. The strongest single correlation in this study was between QLQ-C30 'physical functioning' and albumin level, where the Spearman's rho was 0.40. On the other hand, albumin-to-ALP ratio had the highest number (a total of ten) of potentially clinically important correlations with QOL.

In our earlier study, we have developed C30 and HCC18 index-scores in an attempt to simplify the

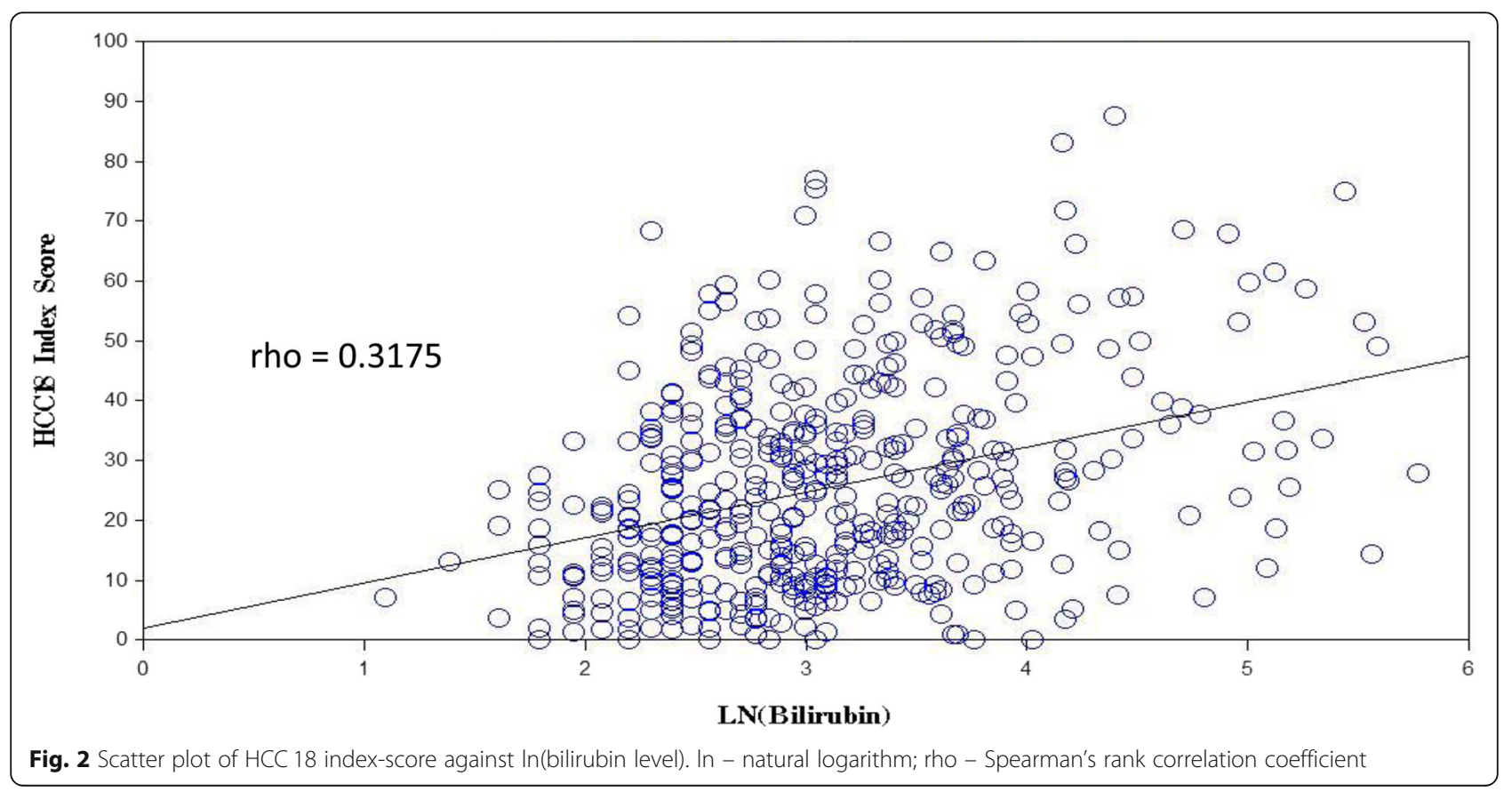




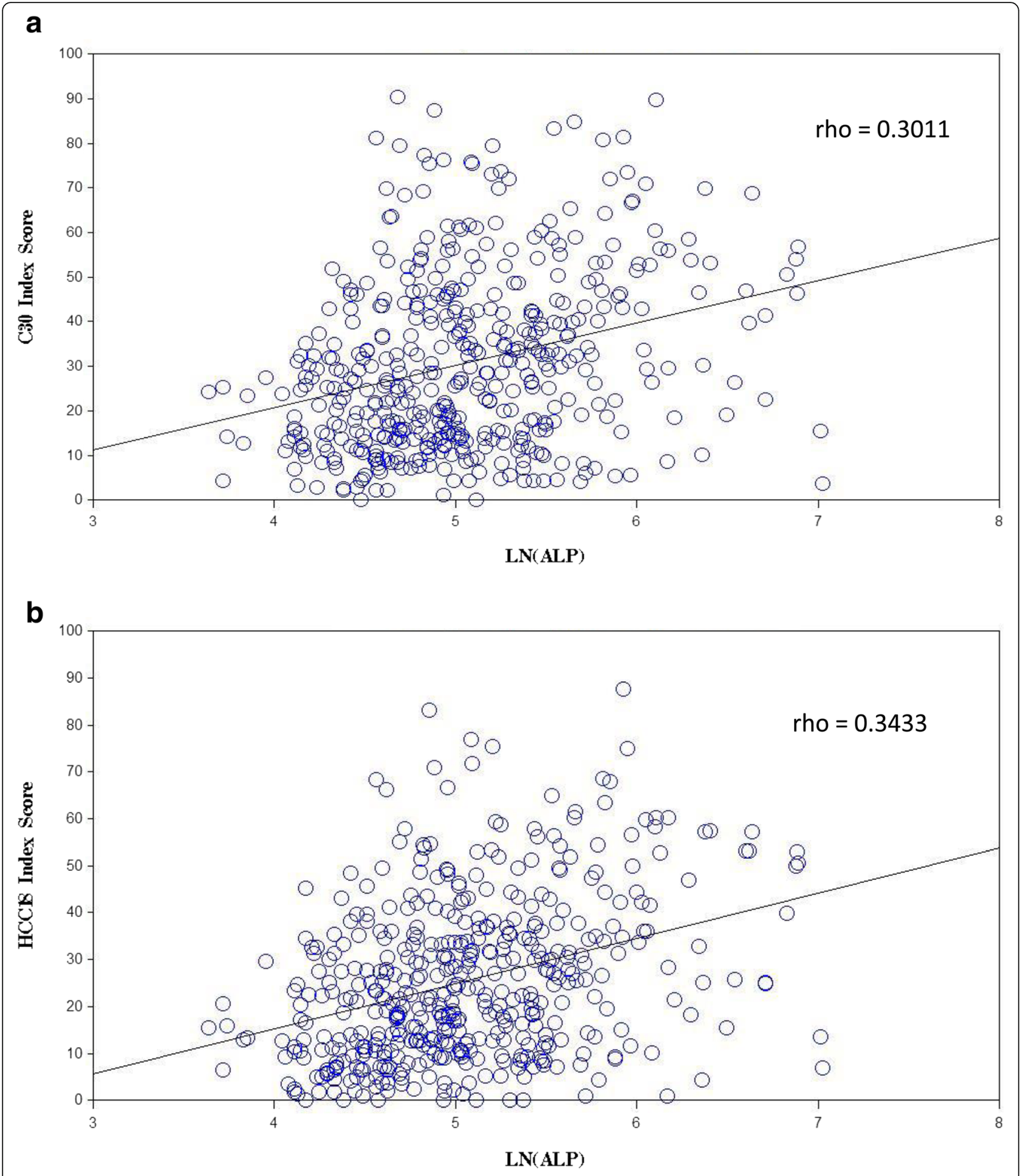

Fig. 3 a. Scatter plot of C30 index-score against In(alkaline phosphatase level). b Scatter plot of HCC1 8 index-score against In(alkaline phosphatase level). In - natural logarithm; rho - Spearman's rank correlation coefficient 


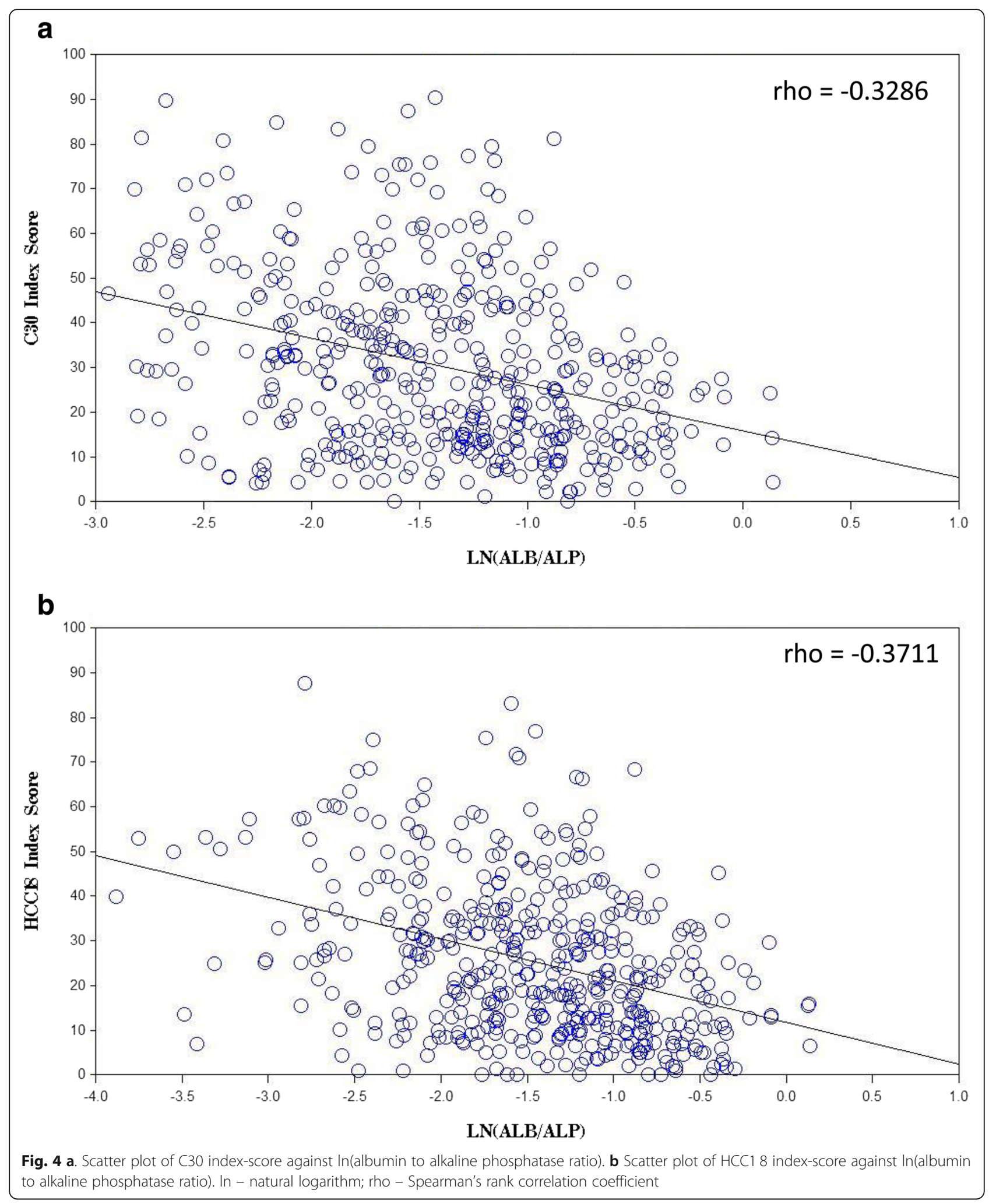


Table 5 Univariate logistic regressions of health related quality of life variables for abnormal categorical liver function variables

\begin{tabular}{|c|c|c|c|c|c|c|c|c|c|c|c|c|}
\hline \multirow[t]{2}{*}{ Variable Name } & \multicolumn{3}{|c|}{ Child's classes B to C } & \multicolumn{3}{|c|}{ ALBI grades 2 to 3} & \multicolumn{3}{|c|}{ Presence of ascites } & \multicolumn{3}{|c|}{ MELD grades 2 to 3} \\
\hline & $\overline{O R}$ & $95 \% \mathrm{Cl}$ & $\overline{p \text {-value }}$ & $\mathrm{OR}$ & $95 \% \mathrm{Cl}$ & $p$-value & $\overline{O R}$ & $95 \% \mathrm{Cl}$ & $p$-value & OR & $95 \% \mathrm{Cl}$ & $p$-value \\
\hline \multicolumn{13}{|l|}{ EORTC QLQ-C30 } \\
\hline Physical functioning & 0.972 & $0.964-0.981$ & $<0.001$ & 0.962 & $0.952-0.973$ & $<0.001$ & 0.974 & $0.965-0.982$ & $<0.001$ & 0.989 & $0.981-0.997$ & 0.009 \\
\hline Role functioning & 0.982 & $0.976-0.987$ & $<0.001$ & 0.975 & $0.967-0.983$ & $<0.001$ & 0.982 & $0.976-0.988$ & $<0.001$ & 0.992 & $0.986-0.998$ & 0.007 \\
\hline Emotional functioning & 0.997 & $0.990-1.005$ & 0.508 & 0.997 & $0.989-1.004$ & 0.396 & 0.995 & $0.988-1.003$ & 0.262 & 1.002 & $0.994-1.010$ & 0.597 \\
\hline Cognitive functioning & 0.988 & $0.980-0.996$ & 0.002 & 0.987 & $0.979-0.996$ & 0.003 & 0.991 & $0.980-1.001$ & 0.082 & 0.993 & $0.985-1.000$ & 0.064 \\
\hline Social functioning & 0.990 & $0.984-0.996$ & 0.002 & 0.986 & $0.979-0.993$ & $<0.001$ & 0.989 & $0.982-0.996$ & 0.001 & 0.995 & $0.989-1.001$ & 0.124 \\
\hline Global quality of life & 0.980 & $0.972-0.987$ & $<0.001$ & 0.978 & $0.970-0.986$ & $<0.001$ & 0.979 & $0.970-0.987$ & $<0.001$ & 0.989 & $0.981-0.996$ & 0.003 \\
\hline Fatigue & 1.022 & $1.015-1.029$ & $<0.001$ & 1.025 & $1.017-1.033$ & $<0.001$ & 1.025 & $1.017-1.032$ & $<0.001$ & 1.010 & $1.003-1.016$ & 0.003 \\
\hline Nausea \& vomiting & 1.014 & $1.005-1.023$ & 0.002 & 1.019 & $1.007-1.031$ & 0.001 & 1.013 & $1.004-1.022$ & 0.004 & 1.009 & $1.001-1.018$ & 0.037 \\
\hline Pain & 1.005 & $0.999-1.011$ & 0.099 & 1.008 & $1.002-1.015$ & 0.010 & 1.007 & $1.000-1.013$ & 0.036 & 0.998 & $0.992-1.004$ & 0.479 \\
\hline Dyspnoea & 1.016 & $1.009-1.022$ & $<0.001$ & 1.016 & $1.009-1.023$ & $<0.001$ & 1.017 & $1.010-1.024$ & $<0.001$ & 1.007 & $1.001-1.013$ & 0.033 \\
\hline Insomnia & 1.009 & $1.004-1.015$ & 0.001 & 1.010 & $1.005-1.016$ & $<0.001$ & 1.010 & $1.005-1.016$ & 0.001 & 0.999 & 0.993-1.004 & 0.638 \\
\hline Appetite loss & 1.014 & $1.008-1.019$ & $<0.001$ & 1.016 & $1.010-1.023$ & $<0.001$ & 1.015 & $1.009-1.020$ & $<0.001$ & 1.007 & $1.002-1.012$ & 0.010 \\
\hline Constipation & 1.004 & $0.998-1.011$ & 0.205 & 1.002 & $0.995-1.010$ & 0.508 & 1.007 & $0.999-1.014$ & 0.072 & 0.998 & $0.991-1.005$ & 0.586 \\
\hline Diarrhea & 1.017 & $1.009-1.024$ & $<0.001$ & 1.016 & $1.007-1.025$ & 0.003 & 1.017 & $1.010-1.025$ & $<0.001$ & 1.012 & $1.005-1.019$ & 0.001 \\
\hline Financial difficulties & 1.003 & $0.998-1.008$ & 0.253 & 1.007 & $1.002-1.012$ & 0.011 & 1.004 & 0.998-1.009 & 0.200 & 1.000 & $0.995-1.006$ & 0.861 \\
\hline \multicolumn{13}{|l|}{ EORTC QLQ-HCC18 } \\
\hline Fatigue & 1.024 & $1.016-1.032$ & $<0.001$ & 1.030 & $1.020-1.040$ & $<0.001$ & 1.022 & $1.013-1.030$ & $<0.001$ & 1.008 & $1.001-1.016$ & 0.027 \\
\hline Body Image & 1.029 & $1.020-1.038$ & $<0.001$ & 1.025 & $1.015-1.035$ & $<0.001$ & 1.034 & $1.024-1.044$ & $<0.001$ & 1.014 & $1.005-1.022$ & 0.001 \\
\hline Jaundice & 1.024 & $1.015-1.033$ & $<0.001$ & 1.011 & $1.002-1.021$ & 0.020 & 1.016 & $1.007-1.025$ & 0.001 & 1.018 & $1.009-1.027$ & $<0.0001$ \\
\hline Nutrition & 1.027 & $1.017-1.037$ & $<0.001$ & 1.026 & $1.015-1.037$ & $<0.001$ & 1.024 & $1.014-1.033$ & $<0.001$ & 1.013 & $1.004-1.022$ & 0.003 \\
\hline Pain & 1.012 & $1.004-1.020$ & 0.003 & 1.013 & $1.004-1.022$ & 0.004 & 1.013 & $1.005-1.022$ & 0.001 & 1.001 & 0.993-1.009 & 0.802 \\
\hline Fever & 1.023 & $1.009-1.037$ & 0.001 & 1.033 & $1.014-1.053$ & 0.001 & 1.012 & $0.999-1.026$ & 0.079 & 1.011 & $0.998-1.025$ & 0.085 \\
\hline Sex life & 1.004 & $0.999-1.010$ & 0.130 & 1.007 & $1.001-1.013$ & 0.017 & 1.002 & $0.997-1.008$ & 0.431 & 1.005 & 0.999-1.010 & 0.089 \\
\hline Abdominal swelling & 1.023 & $1.017-1.029$ & $<0.001$ & 1.019 & $1.012-1.026$ & $<0.001$ & 1.028 & $1.022-1.035$ & $<0.001$ & 1.009 & $1.004-1.015$ & 0.001 \\
\hline \multicolumn{13}{|l|}{ Index-scores } \\
\hline C30 index-score & 1.029 & $1.019-1.040$ & $<0.001$ & 1.037 & $1.024-1.049$ & $<0.001$ & 1.031 & $1.020-1.042$ & $<0.001$ & 1.011 & $1.001-1.021$ & 0.028 \\
\hline HCC18 index-score & 1.045 & $1.032-1.058$ & $<0.001$ & 1.044 & $1.029-1.058$ & $<0.001$ & 1.043 & $1.030-1.056$ & $<0.001$ & 1.020 & $1.009-1.032$ & $<0.001$ \\
\hline
\end{tabular}

$A L B I$ albumin-bilirubin, $\mathrm{Cl}$ Confidence intervals, EORTC European Organization for Research and Treatment of Cancer, MELD Model for End-stage Liver Disease, OR Odds ratio

Table 6 Multivariate logistic regressions of clinical and health related quality of life variables for abnormal categorical liver function variables

\begin{tabular}{|c|c|c|c|c|c|c|c|c|c|c|c|c|}
\hline & \multicolumn{3}{|c|}{ Child's classes B to C } & \multicolumn{3}{|c|}{ ALBI grades 2 to 3} & \multicolumn{3}{|c|}{ Presence of ascites } & \multicolumn{3}{|c|}{ MELD grades 2 to 3} \\
\hline & $\mathrm{OR}$ & $95 \% \mathrm{Cl}$ & $p$-value & $\mathrm{OR}$ & $95 \% \mathrm{Cl}$ & $p$-value & OR & $95 \% \mathrm{Cl}$ & $p$-value & $\mathrm{OR}$ & $95 \% \mathrm{Cl}$ & $p$-value \\
\hline $\begin{array}{l}\text { EORTC QLQ-C30 } \\
\text { Physical Functioning }\end{array}$ & 0.987 & $0.974-1.000$ & 0.045 & 0.973 & $0.961-0.985$ & $<0.001$ & - & - & - & - & - & - \\
\hline $\begin{array}{l}\text { EORTC QLQ-HCC18 } \\
\text { Abdominal Swelling }\end{array}$ & 1.012 & $1.003-1.021$ & 0.011 & - & - & - & 1.020 & $1.012-1.028$ & $<0.001$ & - & - & - \\
\hline CUPI & 4.239 & $2.357-7.626$ & $<0.001$ & 2.580 & $1.557-4.277$ & $<0.001$ & 10.538 & 5.944-18.681 & $<0.001$ & - & - & - \\
\hline Bilirubin & 1.100 & $1.073-1.126$ & $<0.001$ & 1.089 & $1.058-1.121$ & $<0.001$ & 1.010 & $1.001-1.019$ & 0.029 & 1.093 & $1.072-1.114$ & $<0.001$ \\
\hline $\ln (\mathrm{AFP})$ & 0.838 & $0.763-0.920$ & 0.001 & - & - & - & 0.790 & $0.721-0.865$ & $<0.001$ & - & - & - \\
\hline $\begin{array}{l}\text { Planned treatment } \\
\text { modality }\end{array}$ & - & - & - & 2.149 & $1.162-3.972$ & 0.015 & - & - & - & - & - & - \\
\hline
\end{tabular}


various domains and items scores in the EORTC QLQ-C30 and QLQ-HCC18 tools respectively for survival analyses, and they were found to be significant prognostic factors for OS [30]. We proposed to use these 2 respective index-scores as they are easy to calculate and could be conducted in daily clinic setting. In the current analysis, we aimed to assess the correlations between QOL and liver function in HCC patients. Without prior knowledge on the level of correlation with various QOL factors, we have included the available index-scores alongside the 'standard' QOL domains and items in the analyses in order to assess how well each of these could perform. Findings from the current analyses support the fact that the 2 index-scores have potentially clinically important correlations with four continuous liver function variables. None-the-less, it is acknowledged that these indices are by no means able to replace the domains and items within QLQ-C30 and QLQ-HCC18 that addressed QOL in greater depth.

The current study has some limitations. Since the sample size of this study was large enough, even what appeared to be relatively weak correlations have been shown to have statistically significant $p$-values. For examples, a number of QOL factors showed significant correlations with ALT level and ALP-to-platelet ratio. However, it has to be noted that the magnitudes of these Spearman's rho were too weak and thus they are unlikely to have clinical importance. Another limitation would be the lack of follow up assessments of liver function and QOL. These data could be potentially useful, since correlation analyses between QOL and liver function at later time-points may further depict the relationships between liver function and QOL. Thus, further studies with longitudinal follow-up data are warranted.

QOL has gained increasing attention in HCC patient management; specifically, improving patients' QOL has become an important goal to clinicians. Most phase III clinical trials in HCC patients reported QOL as one of the main study endpoints [39-43]. QOL in HCC patients is known to be related to the tumor severity and treatment toxicity. The current study reported potentially clinically important correlations between QOL and liver function in HCC patients. The study findings highlight to clinicians the relevance of liver function in addition to tumor burden to QOL among HCC patients. Along with $\mathrm{HCC}$ tumor per se, liver functional impairment may be the result of other co-existing conditions including viral infections and biliary obstruction. Interventional treatments, by means of anti-viral drug administration and radiological or surgical biliary drainage respectively might improve liver function and have positive impact on QOL of HCC patients. Future trials are warranted to assess whether treatment to enhance liver function could improve HCC patients' QOL.

\section{Conclusions}

With the background knowledge that QOL and liver function are both independent prognostic factors for survival in HCC patients irrespective of stage, this study further explored the relationship between QOL and liver function. In HCC patients, baseline QOL assessment (using EORTC QLQ-C30, QLQ-HCC18, C30 index-score or HCC18 index-score) is significantly correlated with liver function. Based on the findings of this study, future trials are warranted to assess whether treatment to enhance liver function could improve HCC patients' QOL.

\section{Abbreviations \\ AFP: Alpha fetoprotein; ALBI: Albumin-bilirubin; ALP: Alkaline phosphatase; ALT: Alanine transaminase; Cl: 95\% confidence intervals; CLDQ: Chronic Liver Disease Questionnaire; ECOG: Eastern Cooperative Oncology Group; \\ EORTC: European Organization for Research and Treatment of Cancer; FACT- G: Functional Assessment of Cancer Therapy - General; HBV: Hepatitis B virus; HCC: Hepatocellular carcinoma; HCV: Hepatitis C virus; INR: International normalized ratio; MELD: Model for End-stage Liver Disease; OR: Odds ratio; OS: Overall survival; QOL: Quality of life; rho: Spearman's rank correlation coefficient}

\section{Acknowledgements}

We thank all authors for their contributions to the research.

Funding

This study received no funding, grant or financial support.

Availability of data and materials

Availability of dataset for review could be requested.

Authors' contributions

$\mathrm{LL}$ and $\mathrm{WY}$ contributed to this paper with conception and design of the study. LL, WY, EPH, SLC, JK, SCHY and NLST contributed to acquisition of data. FM, LL and WY contributed to data analysis and interpretation of data. All authors contributed to drafting and critical revision and editing, and final approval of the manuscript.

Ethics approval and consent to participate

The study was approved by the Joint Chinese University of Hong Kong New Territories East Cluster Clinical Research Ethics Committee.

All participants to this study understood and signed written consent prior to study inclusion.

Consent for publication

All participants consented to the study consented for publication.

\section{Competing interests}

All authors have no competing interests with regards to the study. No potential conflicts of interest.

\section{Publisher's Note}

Springer Nature remains neutral with regard to jurisdictional claims in published maps and institutional affiliations.

\section{Author details}

'Department of Clinical Oncology, Prince of Wales Hospital, Faculty of Medicine, The Chinese University of Hong Kong, Shatin, NT, Hong Kong SAR. ${ }^{2}$ State Key Laboratory in Oncology in South China, Prince of Wales Hospital, Faculty of Medicine, The Chinese University of Hong Kong, Shatin, Hong Kong SAR. ${ }^{3}$ Department of Chemical Pathology, Li Ka Shing Institute of Health Sciences, Faculty of Medicine, The Chinese University of Hong Kong, Shatin, Hong Kong SAR. ${ }^{4}$ Department of Diagnostic and Interventional Radiology, Prince of Wales Hospital, Shatin, Faculty of Medicine, The Chinese University of Hong Kong, Shatin, Hong Kong SAR. 


\section{Received: 5 May 2018 Accepted: 10 April 2019} Published online: 02 May 2019

\section{References}

1. Ferlay J, Soerjomataram I, Dikshit R, Eser S, Mathers C, Rebelo M, Parkin DM, Forman D, Bray F. Cancer incidence and mortality worldwide: sources, methods and major patterns in GLOBOCAN 2012. Int J Cancer. 2015;136(5):E359-86.

2. Zhang $B H$, Yang BH, Tang ZY. Randomized controlled trial of screening for hepatocellular carcinoma. J Cancer Res Clin Oncol. 2004;130(7):417-22.

3. Kudo M. Japan's successful model of Nationwide hepatocellular carcinoma surveillance highlighting the urgent need for global surveillance. Liver Cancer. 2012;1(3-4):141-3.

4. Llovet JM, Bru C, Bruix J. Prognosis of hepatocellular carcinoma: the BCLC staging classification. Semin Liver Dis. 1999;19(3):329-38.

5. Forner A, Reig ME, de Lope CR, Bruix J. Current strategy for staging and treatment: the BCLC update and future prospects. Semin Liver Dis. 2010;30(1):61-74.

6. Omata M, Cheng AL, Kokudo N, Kudo M, Lee JM, Jia J, Tateishi R, Han $\mathrm{KH}$, Chawla YK, Shiina S, et al. Asia-Pacific clinical practice guidelines on the management of hepatocellular carcinoma: a 2017 update. Hepatol Int. 2017;11(4):317-70.

7. EASL-EORTC clinical practice guidelines. management of hepatocellular carcinoma. J Hepatol. 2012;56(4):908-43.

8. Heimbach JK, Kulik LM, Finn RS, Sirlin CB, Abecassis MM, Roberts LR, Zhu AX, Murad MH, Marrero JA. AASLD guidelines for the treatment of hepatocellular carcinoma. Hepatology. 2018;67(1):358-80.

9. Chin PL, Chu DZ, Clarke KG, Odom-Maryon T, Yen Y, Wagman LD. Ethnic differences in the behavior of hepatocellular carcinoma. Cancer. 1999;85(9):1931-6.

10. Hsu C, Shen YC, Cheng CC, Hu FC, Cheng AL. Geographic difference in survival outcome for advanced hepatocellular carcinoma: implications on future clinical trial design. Contemp Clin Trials. 2010;31(1):55-61.

11. Colombo M, Kuo G, Choo QL, Donato MF, Del Ninno E, Tommasini MA, Dioguardi N, Houghton M. Prevalence of antibodies to hepatitis C virus in Italian patients with hepatocellular carcinoma. Lancet. 1989;2(8670):1006-8.

12. Bruix J, Barrera JM, Calvet X, Ercilla G, Costa J, Sanchez-Tapias JM, Ventura M, Vall M, Bruguera M, Bru C, et al. Prevalence of antibodies to hepatitis C virus in Spanish patients with hepatocellular carcinoma and hepatic cirrhosis. Lancet. 1989;2(8670):1004-6.

13. Davila JA, Morgan RO, Shaib Y, McGlynn KA, El-Serag HB. Hepatitis C infection and the increasing incidence of hepatocellular carcinoma: a population-based study. Gastroenterology. 2004;127(5):1372-80.

14. Ho J, Wu PC, Kung TM. An autopsy study of hepatocellular carcinoma in Hong Kong. Pathology. 1981;13(3):409-16.

15. Liu C, Jia BS, Zou BW, Du H, Yan LN, Yang JY, Jiang L, Wen TF, Lu WS. Neutrophil-to-lymphocyte and aspartate-to-alanine aminotransferase ratios predict hepatocellular carcinoma prognosis after transarterial embolization. Medicine (Baltimore). 2017;96(45):e8512.

16. Fox R, Berhane S, Teng M, Cox T, Tada T, Toyoda H, Kumada T, Kagebayashi C, Satomura S, Johnson PJ. Biomarker-based prognosis in hepatocellular carcinoma: validation and extension of the BALAD model. Br J Cancer. 2014;110(8):2090-8.

17. Schoniger-Hekele M, Muller C, Kutilek M, Oesterreicher C, Ferenci P, Gangl A. Hepatocellular carcinoma in Central Europe: prognostic features and survival. Gut. 2001;48(1):103-9.

18. Wu SJ, Lin YX, Ye H, Xiong XZ, Li FY, Cheng NS. Prognostic value of alkaline phosphatase, gamma-glutamyl transpeptidase and lactate dehydrogenase in hepatocellular carcinoma patients treated with liver resection. Int I Surg 2016:36(Pt A:143-51.

19. Kim HY, Park JW, Joo J, Kim H, Woo SM, Lee WJ, Kim CM. Worse outcome of sorafenib therapy associated with ascites and child-Pugh score in advanced hepatocellular carcinoma. J Gastroenterol Hepatol. 2013;28(11):1756-61.

20. Xiao J, Li G, Lin S, He K, Lai H, Mo X, Chen J, Lin Y. Prognostic factors of hepatocellular carcinoma patients treated by transarterial chemoembolization. Int J Clin Exp Pathol. 2014:7(3):1114-23.

21. Johnson PJ, Berhane S, Kagebayashi C, Satomura S, Teng M, Reeves HL, O'Beirne J, Fox R, Skowronska A, Palmer D, et al. Assessment of liver function in patients with hepatocellular carcinoma: a new evidence-based approach-the ALBI grade. J Clin Oncol. 2015;33(6):550-8.

22. Li MX, Zhao H, Bi XY, Li ZY, Huang Z, Han Y, Zhou JG, Zhao JJ, Zhang YF, Cai JQ. Prognostic value of the albumin-bilirubin grade in patients with hepatocellular carcinoma: validation in a Chinese cohort. Hepatol Res. 2017;47(8):731-41.
23. de Haas RJ, Lim C, Bhangui P, Salloum C, Compagnon P, Feray C, Calderaro J, Luciani A, Azoulay D. Curative salvage liver transplantation in patients with cirrhosis and hepatocellular carcinoma: an intention-to-treat analysis. Hepatology. 2018;67(1):204-15.

24. Yu YQ, Li J, Liao Y, Chen Q, Liao WJ, Huang J. The preoperative alkaline phosphatase-to-platelet ratio index is an independent prognostic factor for hepatocellular carcinoma after hepatic resection. Medicine (Baltimore). 2016;95(51):e5734.

25. Chan AW, Chan SL, Mo FK, Wong GL, Wong W, Cheung YS, Chan HL, Yeo W, Lai PB, To KF. Albumin-to-alkaline phosphatase ratio: a novel prognostic index for hepatocellular carcinoma. Dis Markers. 2015;2015:564057.

26. Li L, Yeo W. Value of quality of life analysis in liver cancer: a clinician's perspective. World J Hepatol. 2017:9(20):867-83.

27. Yeo W, Mo FK, Koh J, Chan AT, Leung T, Hui P, Chan L, Tang A, Lee JJ, Mok TS, et al. Quality of life is predictive of survival in patients with unresectable hepatocellular carcinoma. Ann Oncol. 2006;17(7):1083-9.

28. Diouf M, Filleron T, Barbare JC, Fin L, Picard C, Bouche O, Dahan L, Paoletti $X$, Bonnetain $F$. The added value of quality of life (QoL) for prognosis of overall survival in patients with palliative hepatocellular carcinoma. J Hepatol. 2013;58(3):509-21.

29. Bonnetain F, Paoletti X, Collette S, Doffoel M, Bouche O, Raoul JL, Rougier P, Masskouri F, Barbare JC, Bedenne L. Quality of life as a prognostic factor of overall survival in patients with advanced hepatocellular carcinoma: results from two French clinical trials. Qual Life Res. 2008;17(6):831-43.

30. Li L, Mo FK, Chan SL, Hui EP, Tang NS, Koh J, Leung LK, Poon AN, Hui J, Chu CM, et al. Prognostic values of EORTC QLQ-C30 and QLQ-HCC18 indexscores in patients with hepatocellular carcinoma - clinical application of health-related quality-of-life data. BMC Cancer. 2017;17(1):8.

31. Fielding R, Wong WS. Quality of life as a predictor of cancer survival among Chinese liver and lung cancer patients. Eur J Cancer. 2007;43(11):1723-30.

32. Aaronson NK, Ahmedzai S, Bergman B, Bullinger M, Cull A, Duez NJ, Filiberti A, Flechtner H, Fleishman SB, de Haes JC, et al. The European Organization for Research and Treatment of Cancer QLQ-C30: a qualityof-life instrument for use in international clinical trials in oncology. J Natl Cancer Inst. 1993:85(5):365-76.

33. Blazeby JM, Currie E, Zee BC, Chie WC, Poon RT, Garden OJ. Development of a questionnaire module to supplement the EORTC QLQ-C30 to assess quality of life in patients with hepatocellular carcinoma, the EORTC QLQHCC18. Eur J Cancer. 2004;40(16):2439-44.

34. Panju AH, Danesh A, Minden MD, Kelvin DJ, Alibhai SM. Associations between quality of life, fatigue, and cytokine levels in patients aged 50+ with acute myeloid leukemia. Support Care Cancer. 2009;17(5):539-46.

35. Fung FY, Li M, Breunis H, Timilshina N, Minden MD, Alibhai SM. Correlation between cytokine levels and changes in fatigue and quality of life in patients with acute myeloid leukemia. Leuk Res. 2013;37(3):274-9.

36. Kraemer HC, Morgan GA, Leech NL, Gliner JA, Vaske JJ, Harmon RJ. Measures of clinical significance. J Am Acad Child Adolesc Psychiatry. 2003;42(12):1524-9.

37. Mukaka MM. Statistics corner: a guide to appropriate use of correlation coefficient in medical research. Malawi Med J. 2012;24(3):69-71.

38. Lachin JM. Introduction to sample size determination and power analysis for clinical trials. Control Clin Trials. 1981;2(2):93-113.

39. Llovet JM, Ricci S, Mazzaferro V, Hilgard P, Gane E, Blanc JF, de Oliveira AC, Santoro A, Raoul JL, Forner A, et al. Sorafenib in advanced hepatocellular carcinoma. N Engl J Med. 2008;359(4):378-90.

40. Cheng AL, Kang YK, Chen Z, Tsao CJ, Qin S, Kim JS, Luo R, Feng J, Ye S, Yang TS, et al. Efficacy and safety of sorafenib in patients in the Asia-Pacific region with advanced hepatocellular carcinoma: a phase III randomised, double-blind, placebo-controlled trial. Lancet Oncol. 2009;10(1):25-34.

41. Kudo M, Finn RS, Qin S, Han KH, Ikeda K, Piscaglia F, Baron A, Park JW, Han $\mathrm{G}$, Jassem J, et al. Lenvatinib versus sorafenib in first-line treatment of patients with unresectable hepatocellular carcinoma: a randomised phase 3 non-inferiority trial. Lancet. 2018;391(10126):1163-73.

42. Bruix J, Qin S, Merle P, Granito A, Huang YH, Bodoky G, Pracht M, Yokosuka $\mathrm{O}$, Rosmorduc $\mathrm{O}$, Breder $\mathrm{V}$, et al. Regorafenib for patients with hepatocellular carcinoma who progressed on sorafenib treatment (RESORCE): a randomised, double-blind, placebo-controlled, phase 3 trial. Lancet. 2017;389(10064):56-66.

43. Abou-Alfa GK, Meyer T, Cheng AL, El-Khoueiry AB, Rimassa L, Ryoo BY, Cicin I, Merle P, Chen Y, Park JW, et al. Cabozantinib in patients with advanced and progressing hepatocellular carcinoma. N Engl J Med. 2018;379(1):54-63. 